\title{
Niclosamide repurposed for the treatment of inflammatory airway disease
}

\author{
Inês Cabrita, Roberta Benedetto, Rainer Schreiber, and Karl Kunzelmann \\ Department of Physiology, University of Regensburg, Regensburg, Germany.
}

Inflammatory airway diseases, such as asthma, cystic fibrosis (CF), and chronic obstructive pulmonary disease (COPD), are characterized by mucus hypersecretion and airway plugging. In both CF and asthma, enhanced expression of the $\mathrm{Ca}^{2+}$-activated $\mathrm{Cl}^{-}$channel TMEM16A is detected in mucus-producing club/goblet cells and airway smooth muscle. TMEM16A contributes to mucus hypersecretion and bronchoconstriction, which are both inhibited by blockers of TMEM16A, such as niflumic acid. Here we demonstrate that the FDA-approved drug niclosamide, a potent inhibitor of TMEM16A identified by high-throughput screening, is an inhibitor of both TMEM16A and TMEM16F. In asthmatic mice, niclosamide reduced mucus production and secretion, as well as bronchoconstriction, and showed additional antiinflammatory effects. Using transgenic asthmatic mice, we found evidence that TMEM16A and TMEM16F are required for normal mucus production/secretion, which may be due to their effects on intracellular $\mathrm{Ca}^{2+}$ signaling. TMEM16A and TMEM16F support exocytic release of mucus and inflammatory mediators, both of which are blocked by niclosamide. Thus, inhibition of mucus and cytokine release, bronchorelaxation, and reported antibacterial effects make niclosamide a potentially suitable drug for the treatment of inflammatory airway diseases, such as CF, asthma, and COPD.

Authorship note: IC and RB are cofirst authors.

Conflict of interest: The authors have declared that no conflict of interest exists.

Copyright: () 2019, American Society for Clinical Investigation.

Submitted: February 26, 2019

Accepted: July 2, 2019

Published: August 8, 2019.

Reference information: /CI Insight. 2019;4(15):e128414

https://doi.org/10.1172/jici. insight.128414.

\section{Introduction}

Inflammatory airway diseases, such as cystic fibrosis (CF) $(1,2)$, asthma, and chronic obstructive pulmonary disease (COPD), are characterized by airway obstruction due to mucus hypersecretion, mucus plugging, and bronchoconstriction (3-8). The CF transmembrane conductance regulator (CFTR) $\mathrm{Cl}^{-}$channel is dysfunctional in $\mathrm{CF}$, leading to attenuated fluid and bicarbonate secretion. Along with $\mathrm{Na}^{+}$hyperabsorption and dehydration of the airway surface liquid (ASL; periciliary fluid layer) covering the airway epithelium, this will lead to an inflammatory airway disease $(1,9)$. Although low ASL pH and airway mucus plugging are believed to cause chronic inflammation in $\mathrm{CF}$, mucus hypersecretion in allergic asthma is due to proinflammatory CD4+ Th2-dependent IL-4/IL-13 signaling (10-12). Additional local hypoxia, neutrophilic infiltration, accumulation of reactive oxygen species, and bacterial superinfection cause destructive pulmonary inflammation. It is important to note that asthma-like airway hyperresponsiveness, cough, wheeze, and airway obstruction are also observed in more than $50 \%$ of CF patients, and an inflammation-induced $\beta$-adrenergic hyporesponsiveness is likely to contribute to airway dysfunction in CF (13-15). It is therefore of vital importance to identify novel strategies that limit both mucus production and bronchoconstriction in inflammatory airway disease.

A recent study on adult mice with airway epithelial knockout provided rather surprising results: mice lacking airway epithelial expression of TMEM16A exhibited neither $\mathrm{Ca}^{2+}$-activated TMEM16A nor cAMP-activated CFTR $\mathrm{Cl}^{-}$currents. Yet, the animals did not develop a CF-like phenotype and did not show attenuated mucociliary clearance (16). Normal mouse and human lungs express low levels of TMEM16A. TMEM16A is upregulated in CF and asthma, in both goblet cells and airway smooth muscle. Expression of TMEM16A is also enhanced in arterial smooth muscles in pulmonary arterial hypertension (PAH) (3-8, 17-20). This provides evidence for TMEM16A being important for basal mucus secretion as well as for bronchoconstriction in asthma and CF and for PAH (18, 20-24).

A similar regulatory effect of TMEM16A was found for mucus secretion by intestinal goblet cells, and we described a novel ATP-driven pathway for intestinal mucus secretion (18). TMEM16A supports intestinal fluid secretion and membrane expression of CFTR. Quite surprisingly, the function of TMEM16A in small intestinal epithelial cells, which show very low expression of TMEM16A, seemed to be replaced by 
expression of TMEM16F (25). Taken together, TMEM16A mediates a number of adverse events in inflammatory airway disease, suggesting inhibition rather than activation of TMEM16A as a therapy option in asthma and CF. Using airway- and intestinal-specific knockout mice, we examined the contribution of TMEM16A and the coexpressed homolog TMEM16F to mucus secretion. We found positive effects of niclosamide, a common, FDA-approved drug and potent inhibitor of TMEM16A (21), on various lung parameters. These effects consist of inhibition of mucus release, suppression of inflammation, and airway relaxation, as well as intestinal mucus release. The data support inhibition of TMEM16A and TMEM16F by niclosamide as a potentially novel therapy in inflammatory lung disease.

\section{Results}

Niflumic acid is an inhibitor of TMEM16A/F and blocks airway mucus secretion. OVA-induced allergic asthma in mice caused airway goblet cell metaplasia (Figure 1A). Exposure of asthmatic mice to aerosolized $\mathrm{CCH}$ induced release of intracellular mucus as well as airway contraction (Figure 1, A-C). Pretreatment of OVA-sensitized animals for 3 days with the TMEM16A inhibitor NFA (i.p. and tracheal instillation) before application of $\mathrm{CCH}$ abolished mucus production. Thus, there was no intracellular mucus left to be released by $\mathrm{CCH}$. Moreover, airway contraction was largely inhibited, as judged from airway cross sections. We found that the 2 main TMEM16 paralogs coexpressed in airway epithelial and smooth muscle cells (26), TMEM16A and TMEM16F, were inhibited by NFA, when overexpressed and activated by ionomycin in HEK293 cells (Figure 1, D-F). The data suggest a role of both TMEM16A and TMEM16F for mucus production and contraction of airway smooth muscle (ASM). Although it is entirely possible that NFA also acts via additional cellular mechanisms, it may be beneficial to inhibit both TMEM16 paralogs, as a treatment of inflammatory airway diseases, such as asthma, CF, and COPD (21).

Niclosamide and derivatives - potent inhibitors of TMEM16A/F and $\mathrm{Ca}^{2+}$ signaling. NFA is a nonsteroidal antiinflammatory compound that inhibits goblet cell degranulation and suppresses asthma phenotype (4, 27). A recent high-throughput screen identified the FDA-approved anthelmintic drug niclosamide and related compounds as potent inhibitors of TMEM16A that dilate airways (21). In patch-clamp experiments, we confirmed the inhibitory effect of niclosamide on TMEM16A currents activated by purinergic stimulation in overexpressing HEK293 cells (Figure 2, A-C). Similar to TMEM16A, TMEM16F was also inhibited by niclosamide (Figure 2D). Moreover, niclosamide inhibited TMEM16A expressed endogenously in HT-29 cells, stably transfected with iodide-sensitive yellow fluorescent protein (YFP) (Figure 2E). Apart from niclosamide, other related compounds, such as niclosamide-ETHO, tizoxanide, and nitazoxanide, inhibited endogenous TMEM16A in the nanomolar range and were therefore more potent than the well-known inhibitors CaCCinhAO1 or dichlorophen (Figure 2E). Notably, apart from TMEM16A, HT-29 cells also express TMEM16F (ref. 28 and Figure 2E, inset). We inhibited expression of TMEM16A, TMEM16F, or both TMEM16A/F simultaneously using siRNA. The data suggest that in HT-29 cells ionomycin-induced $\mathrm{Cl}^{-}$currents are mostly due to activation of TMEM16A, with some contribution by TMEM16F. No additional inhibitory effect of niclosamide on YFP quenching was observed in cells with knockout of both TMEM16A and TMEM16F, while niclosamide itself did not induce any significant YFP quenching when applied at different concentrations (Supplemental Figure 1; supplemental material available online with this article; https://doi.org/10.1172/jci.insight.128414DS1).

Previous studies demonstrated that expression of TMEM16 proteins augments intracellular $\mathrm{Ca}^{2+}$ sig- $^{-}$ nals, while the TMEM16 inhibitors CaCCinhAO1 and NFA inhibited intracellular $\mathrm{Ca}^{2+}$ signals (29). Here we demonstrate in different cell types that niclosamide also blocks $\mathrm{Ca}^{2+}$ increase elicited by ATP or by the TMEM16 activator Eact (ref. 30 and Supplemental Figure 2). According to previous studies, TMEM16A augments intracellular $\mathrm{Ca}^{2+}$ signals by increasing filling of the $\mathrm{ER} \mathrm{Ca}^{2+}$ store and by augmenting $\mathrm{IP}_{3}$-induced $\mathrm{Ca}^{2+}$ release and store-operated $\mathrm{Ca}^{2+}$ influx (29). Importantly, niclosamide inhibited $\mathrm{Ca}^{2+}$ signals in freshly isolated goblet cells, which may explain its inhibitory effects on mucus secretion (ref. 18 and Supplemental Figure 2, C and D). Niclosamide inhibited ATP-induced ER Ca ${ }^{2+}$ store release $\left(\mathrm{Ca}^{2+}\right.$ peak) and eliminated $\mathrm{Ca}^{2+}$ influx ( $\mathrm{Ca}^{2+}$ plateau). In $\mathrm{Ca}^{2+}$-free solution ATP-induced store release was fully maintained, while the plateau is eliminated. Niclosamide still attenuated store release but had no further effect on $\mathrm{Ca}^{2+}$ influx in $\mathrm{Ca}^{2+}$-free solution (Supplemental Figure 3).

Niclosamide inhibits mucus production and mucus secretion, ASM contraction, and inflammation. Intracellular mucus in asthmatic mice was reduced by both NFA and niclosamide (Figure 3, A and B). We reported earlier that knockout of TMEM16A inhibits basal mucus secretion in airways and intestine, which results in accumulation of intracellular mucus under control (noninflammatory) conditions (18). According to 


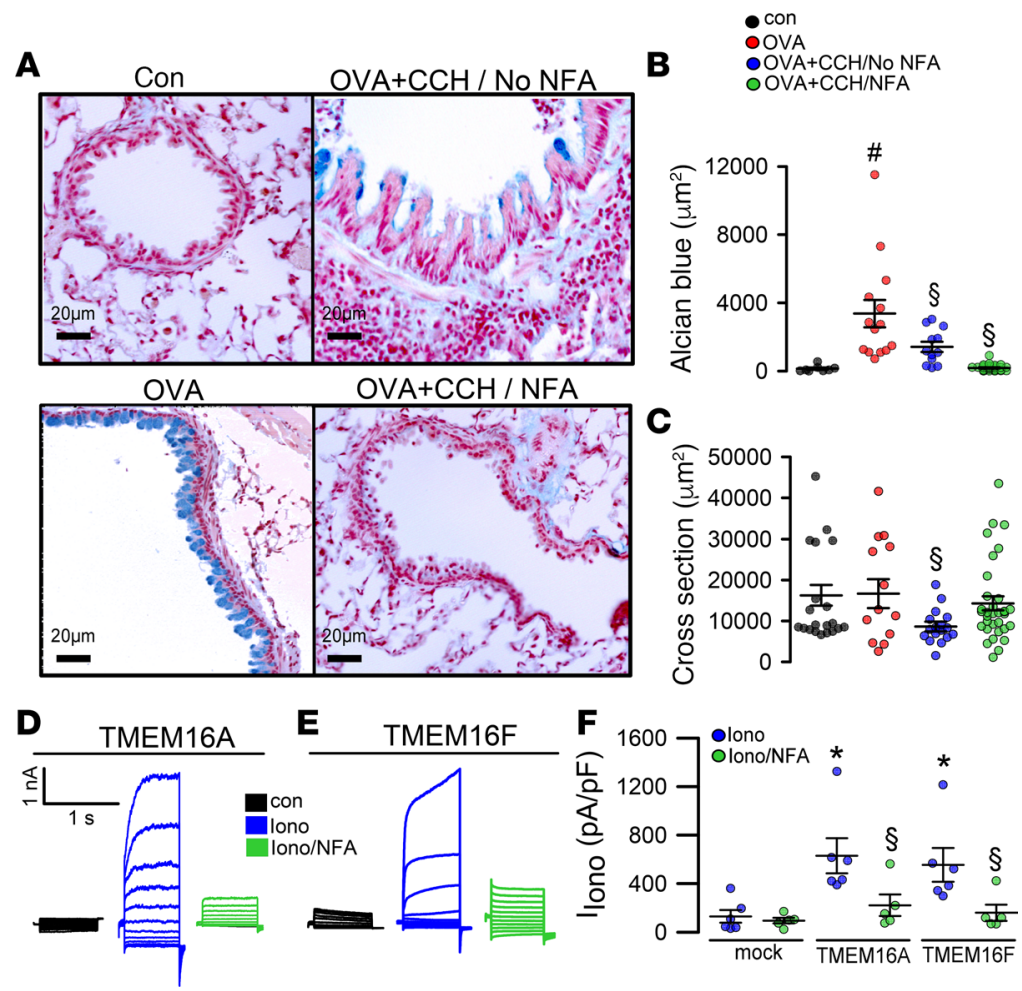

Figure 1. The TMEM16 inhibitor niflumic acid attenuates inflammatory airway disease. (A and B) OVA sensitization induced goblet cell metaplasia as indicated by Alcian blue positivity. Exposure to carbachol (CCH, $25 \mathrm{mg} / \mathrm{mL}$, nebulizer) induced release of mucus and airway contraction ( $n=3$ mice/8-20 airway sections). Scale bars: $20 \mu \mathrm{m}$. Preexposure to the TMEM16A inhibitor niflumic acid (NFA; $0.5 \mathrm{mg} / \mathrm{kg} / \mathrm{d}$, intratracheal application for 3 days) strongly attenuated mucus production and $\mathrm{CCH}$-induced airway contraction (A-C). (C) Cross section of airways indicating airway relaxation by NFA (3 mice/8-13 airway sections). (D-F) Whole-cell currents obtained in patch-clamp experiments with HEK293 cells expressing TMEM16A or TMEM16F. Currents were activated by $1 \mu \mathrm{M}$ ionomycin and inhibited by NFA (20 $\mu$ M) $(n=$ 5-6 cells). Scale bars: $20 \mu \mathrm{m}$. Data are reported as mean \pm SEM. \#Significant increase compared with control $(P<0.05$; unpaired $t$ test); ${ }^{\S}$ significant inhibition $\left(P<0.05\right.$; unpaired $t$ test); ${ }^{*}$ significant activation $\left(P<0.05\right.$; paired $t$ test). $I_{\text {lono' }}$ current induced by ionomycin; $\mathrm{pA} / \mathrm{pF}$, picoAmper per picoFarad.

these earlier studies, TMEM16A/F are expressed in mucus-producing cells, where they exert their positive effects on compartmentalized apical intracellular $\mathrm{Ca}^{2+}$ levels. This supports fusion of mucus-filled granules with the apical membrane, thereby facilitating exocytosis.

However, goblet cell metaplasia and intracellular mucus accumulation under inflammatory (OVA) conditions occurred in both WT (TMEM16 $\left.A^{f / f}\right)$ mice and mice with airway epithelial knockout of TMEM16A (TMEM16A $A^{f l f l}$ FoxJ1-Cre) (Figure 3C). Intracellular mucus was released and airways were contracted after application of $\mathrm{CCH}(\mathrm{OVA} / \mathrm{CCH}$; Figure 3, $\mathrm{C}$ and $\mathrm{G}$ ). In contrast, when mice were treated with niclosamide for 3 days, intracellular mucus was nearly abolished and airway contraction by $\mathrm{CCH}$ was attenuated (OVA/ $\mathrm{CCH} / \mathrm{Niclo}$; Figure 3, C-E). We speculate that niclosamide inhibition of TMEM16A or TMEM16F contributes to inhibition of intracellular mucus production. Notably, the 2 main TMEM16 paralogs expressed in mouse airway epithelial cells, TMEM16A and TMEM16F, along with intracellular TMEM16K (26), were upregulated in OVA-sensitized (asthmatic) mice. Thus, TMEM16A and TMEM16F and possibly TMEM16K may participate in Th2-driven goblet cell metaplasia (Figure 3F and Table 1).

Niclosamide not only reduced mucus production but also reduced cholinergic airway contraction, based on analysis of an airway cross-sectional area (Figure 3, C and G). Potent bronchodilation by niclosamide occurs particularly in cytokine-exposed precontracted airways, which typically show defective $\beta$-adrenergic relaxation (21). Because many CF patients suffer from asthma-like airway hyperresponsiveness (14), niclosamide could also serve as a novel therapeutic tool in CF lung disease. Peribronchial immune cell infiltration observed in allergic airways of OVA-treated animals was strongly reduced by niclosamide and by antiinflammatory NFA (Figure $3 \mathrm{H}$ ). This result suggests an antiinflammatory effect of niclosamide, which corresponds well to inhibition of allergic lung inflammation by the TMEM16A inhibitor benzbromarone $(3,31)$. 
A TMEM16A

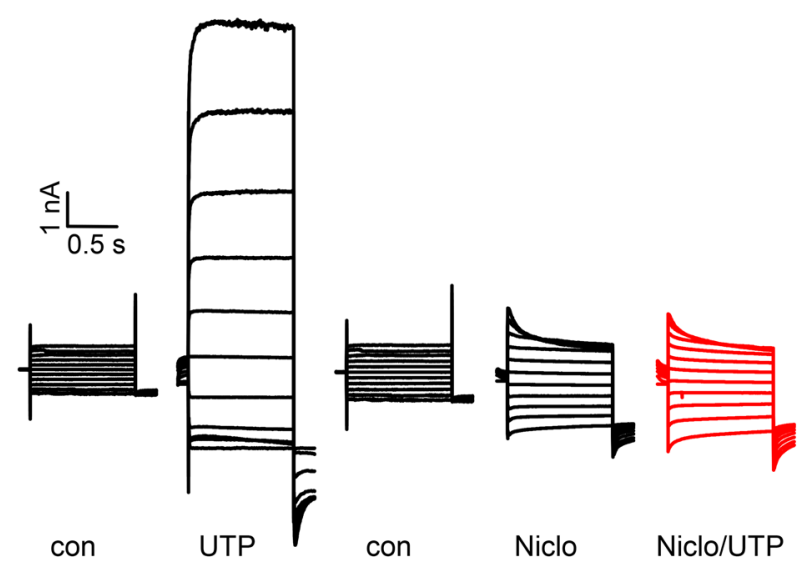

B

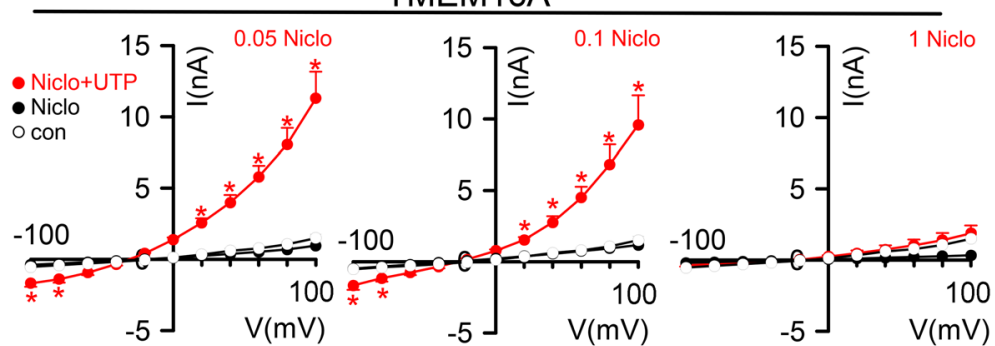

C

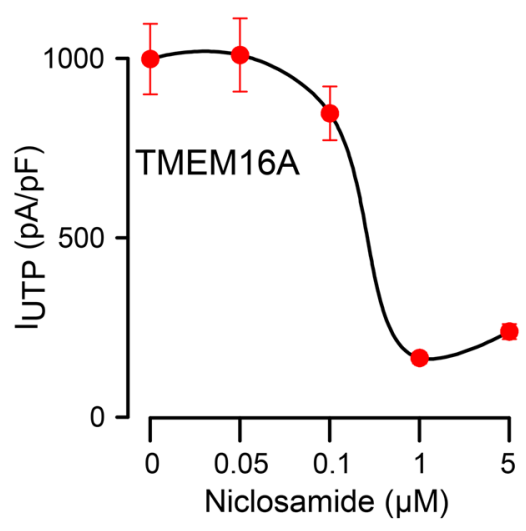

D

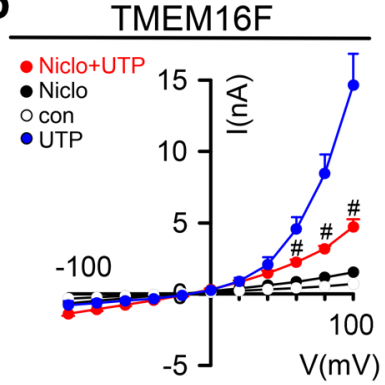

\section{E}
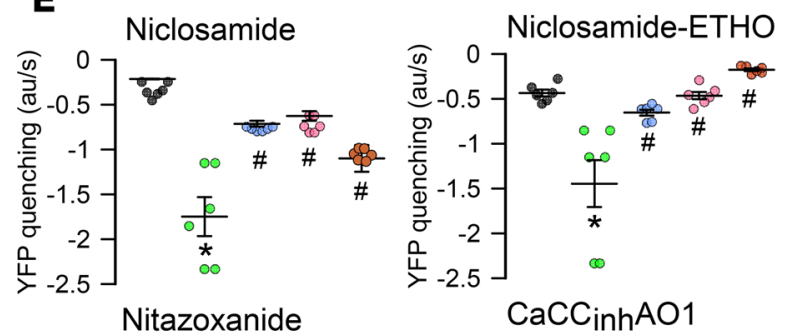

$\mathrm{CaCC}_{\mathrm{inh}} \mathrm{AO} 1$

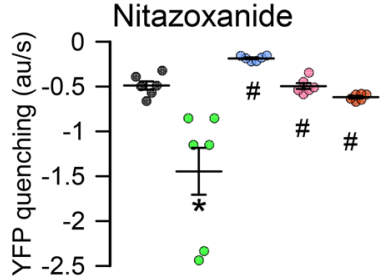

TMEM16A

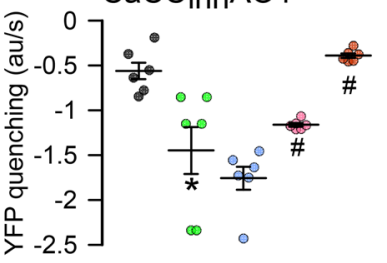

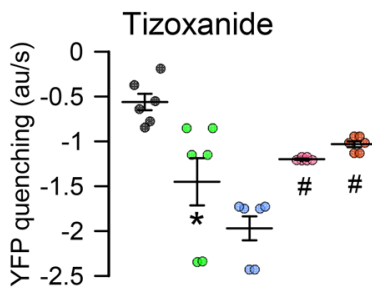

Dichlorophen

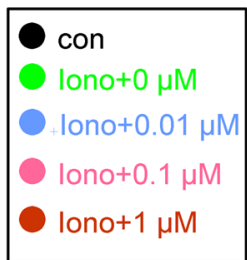

lono+1 $\mu \mathrm{M}$

Figure 2. Inhibition of TMEM16A and TMEM16F by niclosamide. (A) TMEM16A whole-cell currents in TMEM16A-overexpressing HEK293 cells. The purinergic agonist UTP $(100 \mu \mathrm{M})$ was used to activate TMEM16A. UTP-induced currents were inhibited by niclosamide (1 $\mu \mathrm{M})$. (B and C) Concentration-dependent inhibition of TMEM16A by niclosamide ( $n=5-7$ cells). (D) Current/voltage relationship showing inhibition of TMEM16F expressed in HEK293 cells by niclosamide ( $n=5$ cells each). (E) Inhibition of endogenous TMEM16A/F expressed in HT-29 cells examined by iodide quenching. Rate of YFP quenching (AU/s) when applying $20 \mathrm{mM}$ iodide to the extracellular bath solution. HT-29 cells stably overexpressing YFP were stimulated with $1 \mu \mathrm{M}$ ionomycin. Per well, 100,000 cells were seeded ( $n=6-8$ wells for each concentration). Inset: Western blot indicating expression of TMEM16F in HT-29 cells. Data are reported as mean \pm SEM. ${ }^{*}$ Significant activation $(P<0.05$; paired $t$ test); \#significant inhibition $(P<0.05$; unpaired $t$ test).

Niclosamide inhibits inflammation of synoviocytes from patients with rheumatoid arthritis and from a number of other tissues (32). Here, we exposed Calu3 airway epithelial cells to LPS for 48 hours and measured release of the neutrophil attractor IL-8. LPS-induced release of IL- 8 was inhibited in the presence of niclosamide (Figure 4A). Upon stimulation with the Th2 cytokine IL-13, Calu3 cells produced MUC5AC (Figure 4, B and C). IL-13-induced synthesis of MUC5AC was suppressed by knockdown of TMEM16F or treatment of the cells with niclosamide, while niclosamide did not affect expression of TMEM16A or TMEM16F (Figure 4, B-E). Expression of MUC5B was not detected in Calu3 cells. Moreover, we found 
A

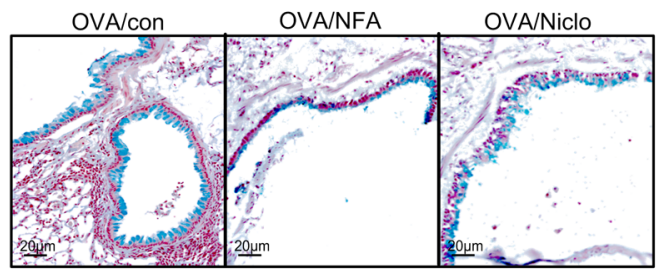

B

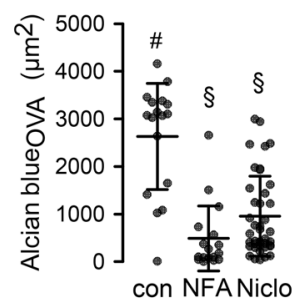

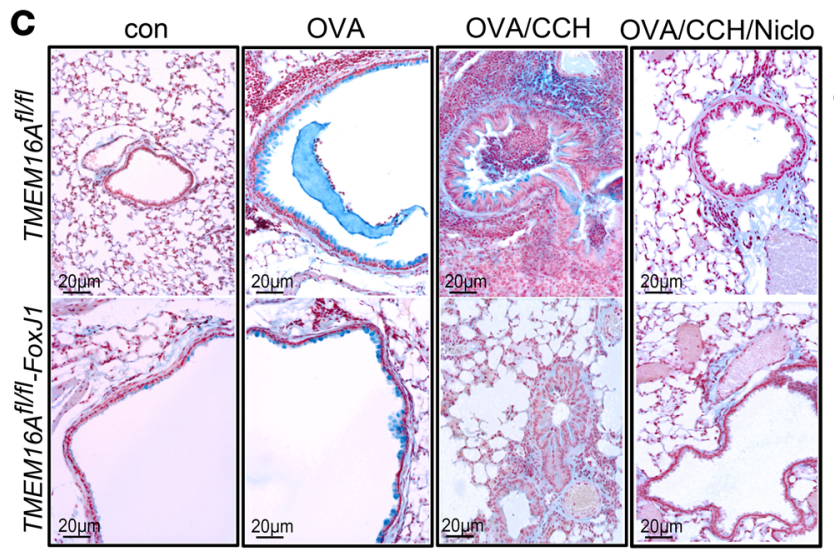

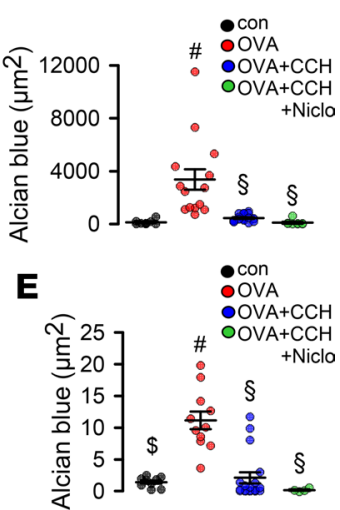

$\mathbf{F}$

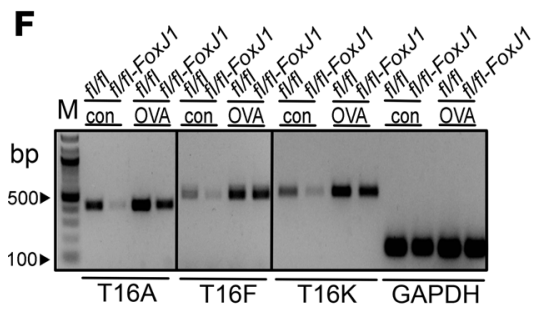

$\mathbf{G}$

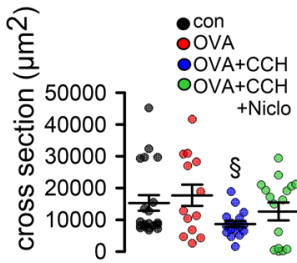

\section{H}

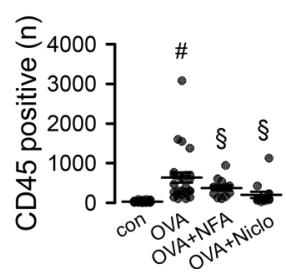

Figure 3. Niclosamide attenuates inflammatory airway disease. (A and B) Goblet cell metaplasia in OVA-sensitized mice detected by Alcian blue. Tracheal instillation of NFA $(0.5 \mathrm{mg} / \mathrm{kg} / \mathrm{d})$ or niclosamide $(13 \mathrm{mg} / \mathrm{kg} / \mathrm{d})$ significantly reduced mucus production ( $n=3$ mice/17-40 airway sections). Scale bars: $15 \mu \mathrm{m}$. (C-E) OVA sensitization of TMEM16A fl/f (fl/fl) and TMEM16A $A^{f / f l}$ Fox/1-Cre (fl/fl-Fox/1) mice induced goblet cell metaplasia. Exposure of $f l / f l$ and fl/fl-Fox]1 mice to CCH (25 $\mathrm{mg} / \mathrm{mL}$, nebulizer) induced mucus release and airway contraction. Preexposure to niclosamide by intratracheal application for 3 days attenuated mucus production and CCH-induced airway contraction ( $n=3$ mice analyzed/4-19 airway sections examined). Scale bars: $20 \mu \mathrm{m}$. (F) Semiquantitative reverse transcription PCR (RT-PCR) analysis of expression of the 3 main TMEM16 paralogs (A, $F$, and $K$ ) in mouse airways, before and after OVA sensitization. TMEM16A, $-F$, and -K were knocked out in ciliated (fl/fl-Fox/1) airway epithelial cells. WT littermates are shown for control (fl/fl). Induction of asthma by OVA treatment caused goblet cell metaplasia and upregulation of transcription of TMEM16A, $-F$, and -K in mucus-producing cells. Lanes were run on the same gel but were noncontiguous. Primers used are listed in Table 2. Quantification is provided in Table 1. (C) Airway cross section indicating airway contraction by muscarinic stimulation (aerosol) and inhibition of contraction by niclosamide ( $n=3$ mice analyzed/13-20 airways sections examined). (H) Number of CD45 ${ }^{+}$cells (airways) under different conditions ( $n=3$ mice/19-20 airway sections). Data are reported as mean \pm SEM. "Significant

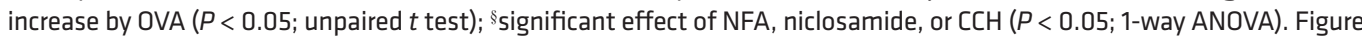
$1, B$ and $C$, and $D$ and $E$ share the same control groups (control, OVA).

that knockdown of both TMEM16A and TMEM16F reduced expression of MUC5AC, and simultaneous knockdown almost abolished MUC5AC expression. Notably, knockdown of TMEM16K also exerted some inhibitory effects on MUC5AC expression (Supplemental Figure 4). The data suggest Th2/IL-13dependent upregulation of TMEM16A, -F, and $-\mathrm{K}$ in airways of asthmatic mice. Although it is likely that niclosamide has additional cellular effects, inhibition of mucus production, bronchoconstriction, and airway inflammation may occur through blockade of TMEM16 paralogs expressed in airway epithelial cells.

Airway epithelial knockout of TMEM16F attenuates mucus production. The data suggest that TMEM16F is relevant for mucus production. In a previous study, we found that knockout of TMEM16A in FoxJ1-positive cells led to an accumulation of mucus due to a lack of mucus release by secretory cells, probably through a paracrine loop (18). KO of TMEM16A may compromise release of IL-6 or other cytokines 
Table 1. Upregulation of expression of TMEM16A, -F, and -K in airways of asthmatic mice

\begin{tabular}{|c|c|c|c|}
\hline \multirow{2}{*}{ TMEM16 } & \multirow{2}{*}{ Mouse } & \multicolumn{2}{|c|}{ Relative expression } \\
\hline & & Control & Asthmatic \\
\hline \multirow[t]{2}{*}{ TMEM16A } & $f I / f I$ & $0.61 \pm 0.06$ & $1.09 \pm 0.11^{A}$ \\
\hline & $f I / f l-F o x / 1$ & $0.07 \pm 0.03$ & $0.51 \pm 0.04^{A}$ \\
\hline \multirow[t]{2}{*}{ TMEM16F } & $f l / f l$ & $0.26 \pm 0.01$ & $0.50 \pm 0.04^{A}$ \\
\hline & $f l / f l-F o x / 1$ & $0.16 \pm 0.02$ & $0.49 \pm 0.07^{A}$ \\
\hline \multirow[t]{2}{*}{ TMEM16K } & $f l / f l$ & $0.40 \pm 0.02$ & $0.77 \pm 0.09^{A}$ \\
\hline & $f l / f l-F o x / 1$ & $0.17 \pm 0.01$ & $0.67 \pm 0.08^{A}$ \\
\hline
\end{tabular}

Summary from semiquantitative RT-PCR shown in Figure 3F. The 3 main TMEM16 paralogs expressed in airway epithelial cells are TMEM16A, TMEM16F, and TMEM16K. Data from mice with knockout of TMEM16A in ciliated epithelial cells (fI/fl-Fox 11 ) and WT littermates $(f l / f I)$. Expression was analyzed in control mice and in OVA-sensitized (asthmatic) mice. Expression of TMEM16A, TMEM16F, and TMEM16K was upregulated in asthmatic mice $(n=4$ reactions each). Bands for TMEM16 and internal GAPDH standard were analyzed densitometrically and the ratio was calculated. Data are reported as mean \pm SEM. ${ }^{A}$ Significant upregulation in asthmatic mice $(P<0.05$; unpaired $t$ test).

that stimulate mucus production (33). However, TMEM16A is also directly involved in mucus secretion by airway epithelial and intestinal goblet cells (18). We generated an airway-specific knockout of

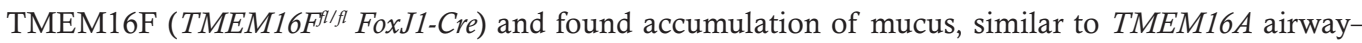
knockout mice (ref. 18 and Figure 5). OVA sensitization induced goblet cell metaplasia, and intracellular mucus was attenuated in TMEM16F ${ }^{q / f l}$ FoxJ1-Cre mice, while cholinergic release of mucus appeared uncompromised (Figure 5).

TMEM16F is required for acute ATP-induced mucus secretion in the intestine. We examined whether TMEM16F has a role for acutely stimulated intestinal mucus secretion, similar to that reported recently for TMEM16A (18). We induced acute mucus release in ex vivo-perfused colon of mice with intestinal knock-

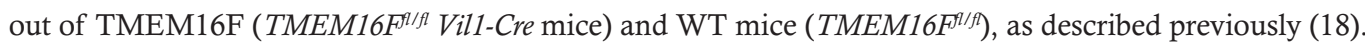
Acute application of methacholine (MCh) induced mucus release in both WT and TMEM16F-knockout intestine, as shown by attenuated periodic acid-Schiff (PAS) staining of intracellular mucus after MCh (Figure 6). Mucus release induced by luminal ATP was somewhat reduced in intestine from TMEM16F-knockout mice (Figure 6). We noticed elongated crypts in knockout animals. After ATP-induced mucus release, the empty granules appeared expanded. These changes could be related to the partial secretory defect but may also be due to the lack of volume regulation properties of TMEM16F (34). Notably, in mice with an intestinal epithelial knockout of TMEM16K, we also noticed a change in tissue architecture (35).

Niclosamide blocks acute intestinal mucus secretion and inhibits $\mathrm{Ca}^{2+}$ signaling. We examined whether niclosamide inhibits intestinal mucus secretion using in vitro-perfused intestinal segments, as described earlier (18). We collected mucus released upon stimulation with MCh or luminal ATP. Intestinal mucus release was quantified in the absence or presence of niclosamide. The amount of mucus MCh released was not affected by niclosamide, while ATP-released mucus was attenuated (Figure 7A). Niclosamide was also applied in vivo for 3 days by i.p. injection or per gavage (Figure 7, E-G). Both i.p. and oral application of niclosamide abolished ATP-induced release of mucus. Although ATP released no mucus, mucus accumulation in intestinal goblet cells was not observed, which may suggest inhibition of both production and secretion of mucus by niclosamide (Figure 7, B-E).

It is rather likely that inhibition of mucus by niclosamide is due to inhibition of TMEM16F for the following reasons: (a) NFA, CaCCinhAO1, and niclosamide blocked all TMEM16F and mucus production; (b) knockout of TMEM16F (TMEM16F ${ }^{\text {q/fl }}$ FoxJ1-Cre) resulted in reduced mucus in asthmatic mice (Figure 5); (c) in Calu3 human airway epithelial cells, knockout of TMEM16F strongly reduced IL-13-induced mucus production (Figure 3 and Supplemental Figure 4); and (d) after inhibition of mucus production by knockdown of TMEM16F, niclosamide had no further effect on IL-13-induced mucus production.

We earlier reported the role of TMEM16F for intracellular $\mathrm{Ca}^{2+}$ signaling (29). In the present study, we confirm these earlier findings because we found reduced $\mathrm{Ca}^{2+}$ increase upon ATP stimulation of freshly isolated crypt cells from TMEM16F $F^{f / f l}$ Vil1-Cre mice (Supplemental Figure 5, A-C). In contrast, CCH-induced $\mathrm{Ca}^{2+}$ rise was not affected (Supplemental Figure 5, D and G). Niclosamide also inhibited ATP-induced $\mathrm{Ca}^{2+}$ increase 

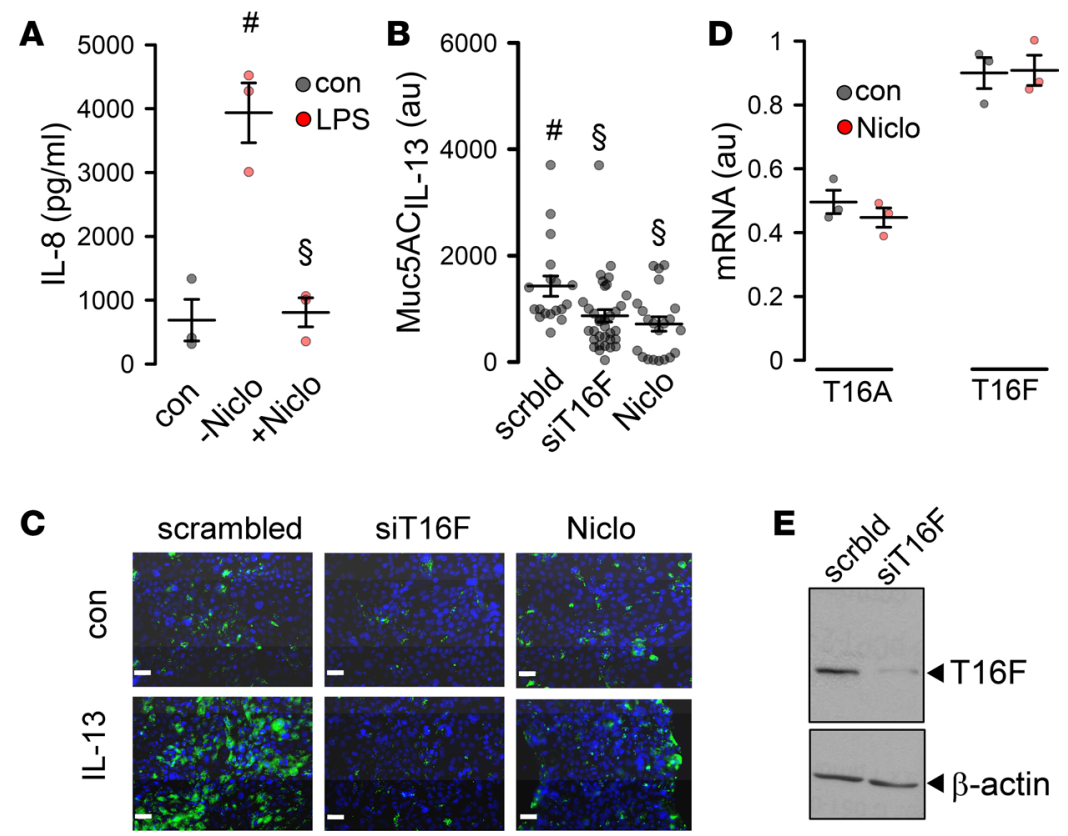

Figure 4. Niclosamide attenuates inflammatory airway response. (A) Effect of niclosamide $(1 \mu \mathrm{M})$ on LPS-induced (10 $\mu \mathrm{g} / \mathrm{mL}$ ) release of IL- 8 in Calu3 cells $(n=3)$. (B and C) IL-13-induced MUC5AC release and inhibition by siRNA TMEM16F and niclosamide $(1 \mu \mathrm{M})(n=19-34$ sections). Scale bars: $15 \mu \mathrm{m}$. Blue color: cell nucleus staining. Green: MUC5AC staining . (D) Effect of niclosamide on mRNA expression for TMEM16A and TMEM16F (semiquantitative RT-PCR) $(n=3)$. (E) Western blot indicating knockdown of TMEM16F by siRNA. The knockdown was $91 \% \pm 4.2 \%(n=3)$. Scale bars: $20 \mu \mathrm{m}$. Data are reported as mean \pm SEM. \#Significant increase (ANOVA); §significant inhibition (ANOVA).

(Supplemental Figure 5E). Comparable results were obtained in goblet cells from small and large intestine. The fluorescence dye FM4-64 can be used to demonstrate acute membrane exocytosis $(18,36)$. We found that stimulation of purinergic receptors by ATP in P2Y2R/TMEM16F-coexpressing cells augmented exocytosis, while cholinergic signaling (CCH in M3R/TMEM16F-coexpressing cells) did not. This result may indicate functional coupling of TMEM16F with purinergic, but not with cholinergic, receptors (Supplemental Figure 6). Taken together, TMEM16A/F support airway and intestinal mucus production and secretion. Niclosamide is an inhibitor of both TMEM16 paralogs. By blocking TMEM16A/F and possibly by additional cellular effects, niclosamide inhibits mucus production/secretion, release of cytokines, and bronchoconstriction.

\section{Discussion}

Role of TMEM16A/F in inflammatory airway diseases. This paper discusses the importance of TMEM16A/F for mucus production and secretion and identifies TMEM16A/F as targets of the anthelmintic drug niclosamide (21). Nevertheless, it is entirely possible that niclosamide acts via additional mechanisms, which are independent of TMEM16A/F $(37,38)$. We recently provided evidence that TMEM16A and TMEM16F can replace each other in exerting their proexocytic effects (25). Unlike TMEM16F, TMEM16A does not transport phospholipids and operates as a $\mathrm{Cl}^{-}$-selective ion channel (39). Yet, both paralogs support intracellular compartmentalized $\mathrm{Ca}^{2+}$ signals (29), and a similar role has been found recently for TMEM16H (40). To further rule out additional effects of niclosamide, a double knockout of TMEM16A/F in mice would have been very useful. However, as described earlier, intestinal-specific double knockout for TMEM16A/F appears to be developmentally lethal (25).

Taken together, we propose niclosamide to be repurposed as a pharmacological tool to treat diseases with excessive mucus secretion, such CF, asthma, and COPD. Notably, antiinflammatory talniflumate, a phthalidyl ester of the TMEM16A/F inhibitor NFA, was shown to improve survival in a CF mouse model of DIOS (41).

Mucus hypersecretion in airways results in mucus plugging, causing reduced mucociliary clearance, the predominant problem in CF lung disease (5). Pronounced upregulation of TMEM16A, particularly in secretory cells, is observed in asthma and CF, but the role of TMEM16A is still unclear $(19-21,42,43)$. The current and previous data present evidence that both TMEM16A and TMEM16F are upregulated during 
A
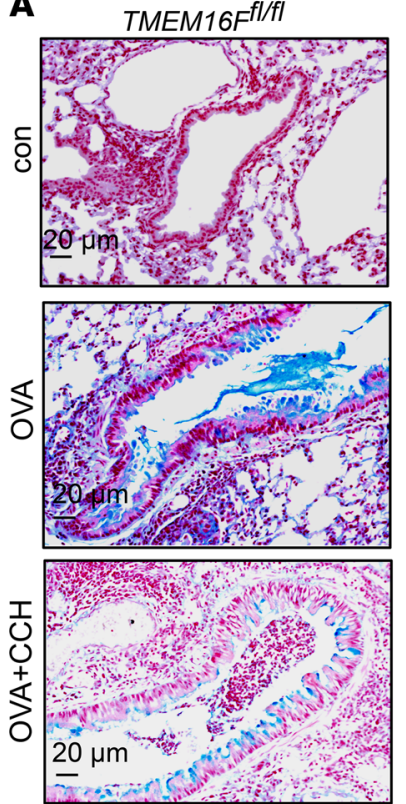

TMEM16F FI/fI-FoxJ1
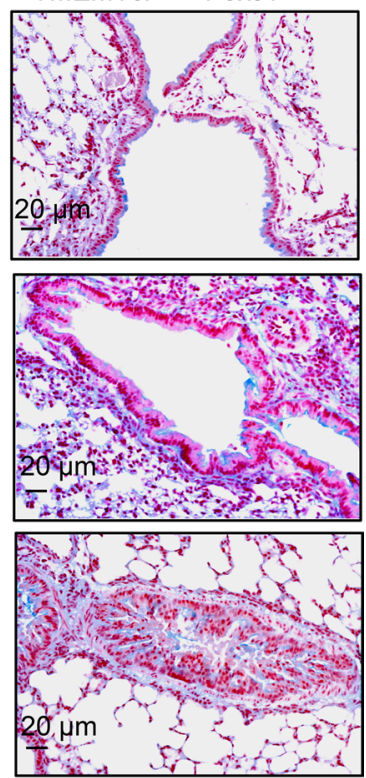

Figure 5. TMEM16F controls mucus production. (A) Mucus in TMEM16Fflff $(f l / f l)$ and TMEM16F Fl/fl Fox/1-Cre (fl/fl-Fox/1) airways under control conditions, after OVA sensitization, and after exposure to $\mathrm{CCH}(25 \mathrm{mg} / \mathrm{mL}$, nebulizer). Scale bars: $20 \mu \mathrm{m}$. (B) Summary Alcian blue staining in $\mathrm{fl} / \mathrm{fl}$ and $\mathrm{fl} / \mathrm{fl}$-Fox/1 animals ( $n=3$ mice/ 8-52 airway sections analyzed). Data are reported as mean \pm SEM. \#Significant increase by OVA (ANOVA); §significant decrease by CCH (ANOVA); \$significant difference from $\mathrm{fl} / \mathrm{fl}$ (ANOVA).

B

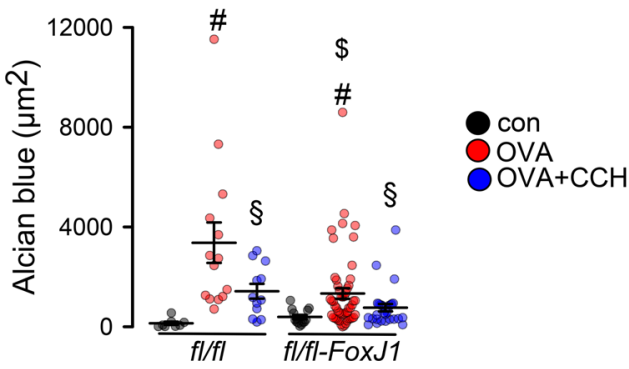

airway inflammation and support mucus secretion, probably by augmenting intracellular $\mathrm{Ca}^{2+}$ signals. Given the potential role of TMEM16A for mucociliary clearance, it may be possible that inhibition by niclosamide reduces mucociliary clearance. However, in preliminary experiments we did not detect a negative effect of niclosamide on stroke amplitude or particle transport in excised mouse tracheas (data not shown).

Using T16AinhAO1 inhibitor, regulation of MUC5AC expression by TMEM16A via STAT6/ ERK1/2 has been proposed (44). However, TMEM16A inhibitors, including T16AinhAO1, are not specific for TMEM16A and inhibit other TMEM16 proteins as well $(21,34)$. The data shown here suggest a role of TMEM16F for mucus production in airways and intestines. Like TMEM16A, TMEM16F is upregulated during Th2-dependent goblet cell metaplasia, along with TMEM16K (Figure 3F). They are the main TMEM16 paralogs expressed in human and mouse airway epithelium $(26,45)$ and are inhibited by common TMEM16A inhibitors, such as NPPB, NS3728, T16inhAO1, and NFA $(34,46)$.

TMEM16A/F control intracellular $\mathrm{Ca}^{2+}$ signals and exocytosis. TMEM16A, -F, and $-\mathrm{K}$ augment intracellular $\mathrm{Ca}^{2+}$ signals induced by stimulation of $\mathrm{G}$ protein-coupled receptors (GPCRs). This is shown in airways, intestines, and kidneys, as well as macrophages, sensory neurons, goblet cells, and different cell lines (16, 29, 35, 47-50). TMEM16 proteins enhance GPCR-induced $\mathrm{Ca}^{2+}$ signals by tethering the ER to the plasma membrane, by $\mathrm{Ca}^{2+}$ influx through TMEM16F, and by refilling the ER $\mathrm{Ca}^{2+}$ store $(29,51)$. ER tethering has also been shown for TMEM16H (40). TMEM16 paralogs may also enhance $\mathrm{Ca}^{2+}$ signals by increasing phosphatidylinositol 4,5-bisphosphate in the plasma membrane (52). Increase in intracellular $\mathrm{Ca}^{2+}$ causes exocytosis of mucus from secretory cells and supports membrane insertion and activation of CFTR $(16,18,52)$.

Inhibition of TMEM16 may improve CF lung disease. Pharmacological activation of TMEM16A and induction of $\mathrm{Cl}^{-}$secretion is thought to improve $\mathrm{CF}$ lung disease (53). However, it is worth considering that in $\mathrm{CF}$, activation of $\mathrm{Cl}^{-}$secretion through stimulation of TMEM16A might be ineffective. Maturation/ function of TMEM16A in ciliated cells is compromised in $\mathrm{CF}(16,54)$, and purinergic continuous $\mathrm{Cl}^{-}$ secretion is essentially through CFTR and not through transient TMEM16A currents $(16,55,56)$. Along 

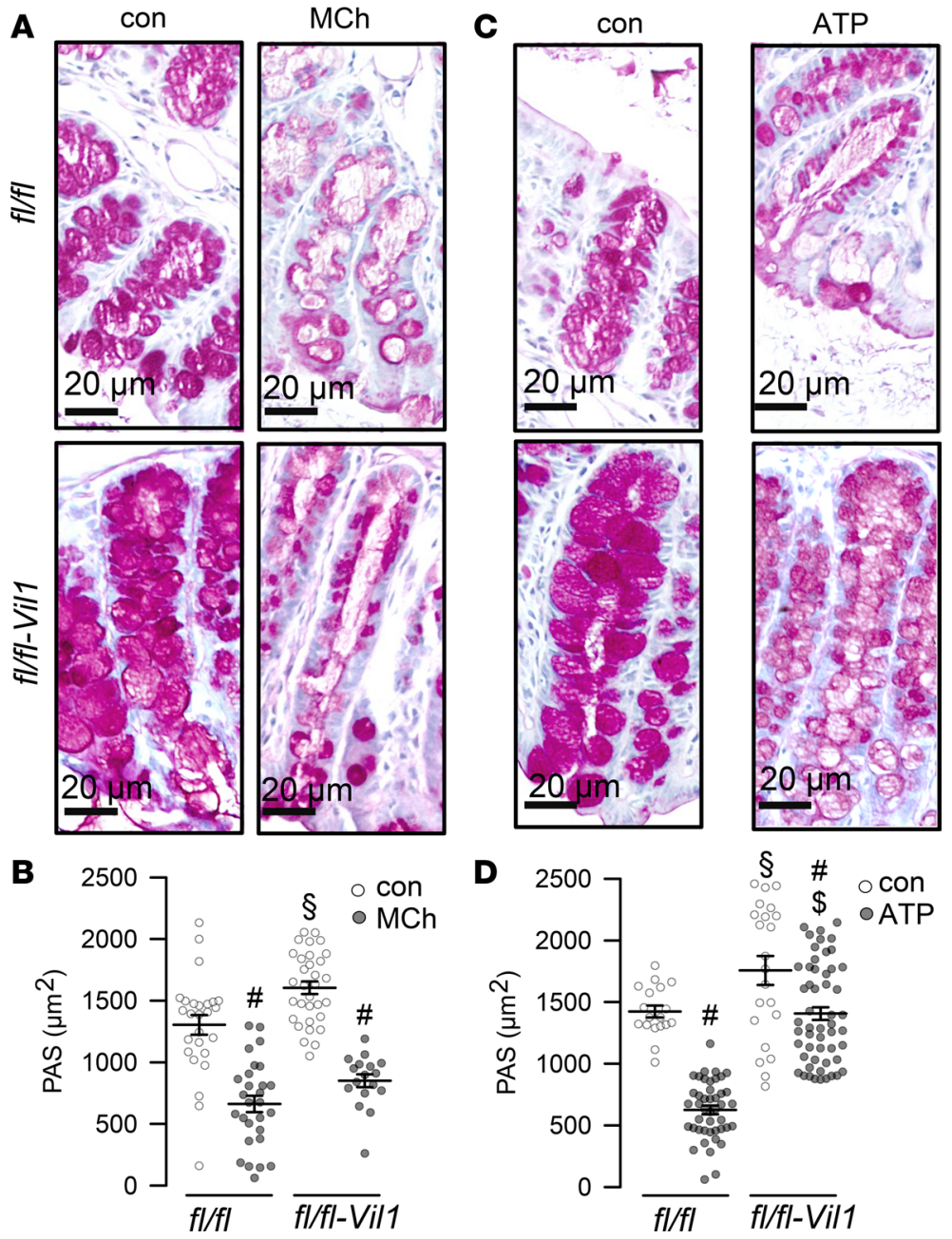

Figure 6. Production of mucus but not release is affected in TMEM16Ffl/fl Vil1-Cre intestine. PAS staining of proximal colon before and after stimulation with methacholine (MCh; $100 \mu \mathrm{M})$ (A and B) ( $n=3$ mice/17-32 sections) or ATP (100 $\mu \mathrm{M})(n=3$ mice $/ 28-85$ sections) (C and D). Scale bars: $20 \mu \mathrm{m}$. Data are reported as mean \pm SEM. \#Significant decrease (ANOVA); §significant increase in fl/fl-Vill (ANOVA); \$significant difference from fl/fl (ANOVA).

this line, earlier trials using stabilized purinergic ligands (denufosol) to restore $\mathrm{Ca}^{2+}$-dependent $\mathrm{Cl}^{-}$secretion failed to demonstrate any benefit in $\mathrm{CF}(57,58)$.

Using niclosamide as a potent inhibitor of TMEM16A/F may have a number of beneficial effects in CF: (a) it is a potent inhibitor of excessive mucus secretion, as shown in the present study; (b) it relaxes airways and thereby reduces bronchoconstriction (21) (and present study); (c) it is a commonly used FDA-approved drug that is well tolerated (37); (d) it was shown to inhibit Pseudomonas aeruginosa quorum sensing (59); (e) inhalable formulations of niclosamide have been developed for the treatment of Pseudomonas lung infections (60); (f) it was shown to have broad antimicrobial effects directed against hospital-acquired bacterial infections (61); (g) it is antiinflammatory (62) (and present study); and (h) it has additional anticancer effects (63). Because niclosamide inhibits mucus release and mucus production, it could be beneficial in interrupting a status asthmaticus or acute CF exacerbation. Taken together, with these reported positive effects, additional preclinical studies and subsequent clinical pilot studies appear indicated.

\section{Methods}

Animals, OVA-induced asthma. All animal experiments complied with the Animal Research: Reporting of In Vivo Experiments (ARRIVE) guidelines and were carried out in accordance with the UK Animals (Scientific Procedures) Act 1986 and associated guidelines, as well as EU Directive 2010/63/EU for animal experiments. Mice (C57BL/6) with a floxed TMEM16A allele and FoxJ1-Cre mice were provided 

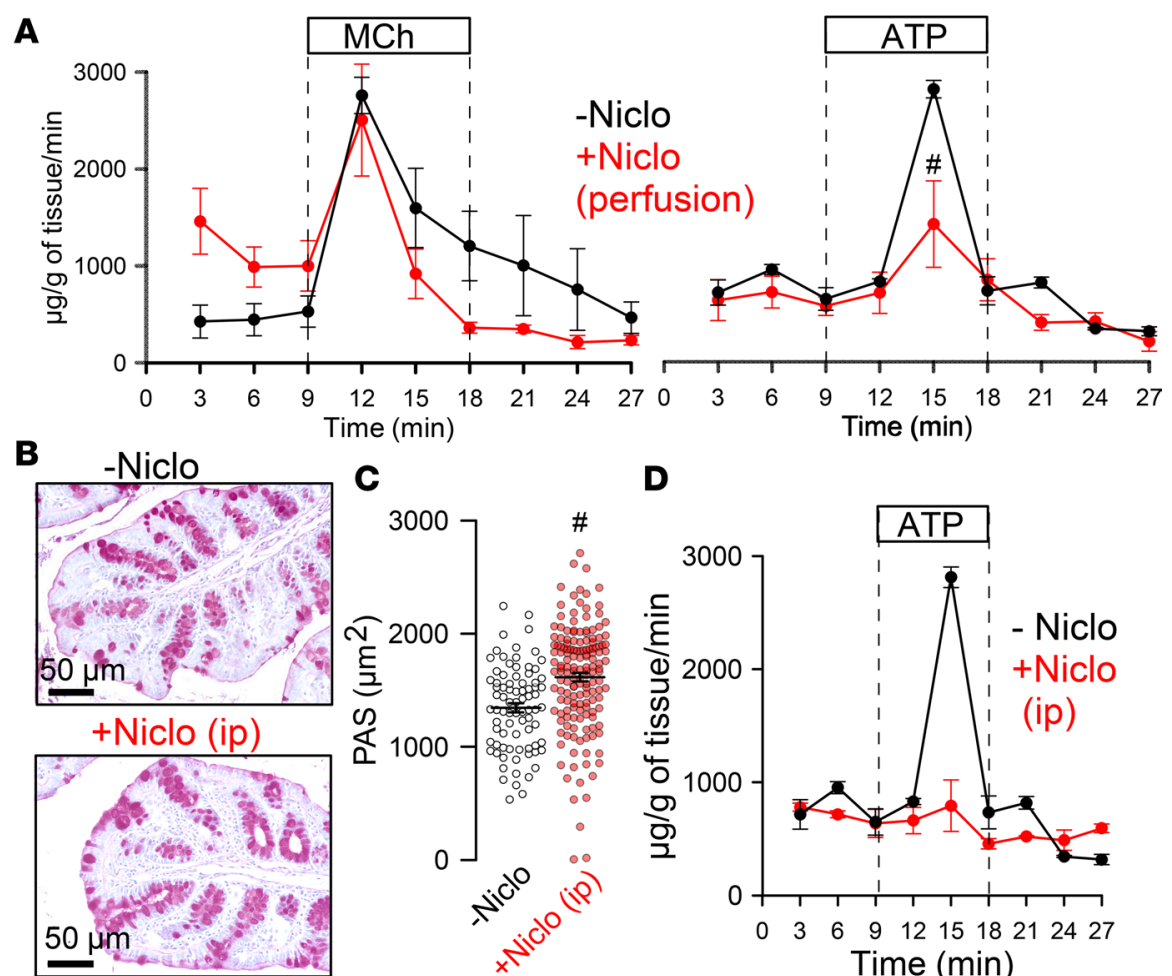

D
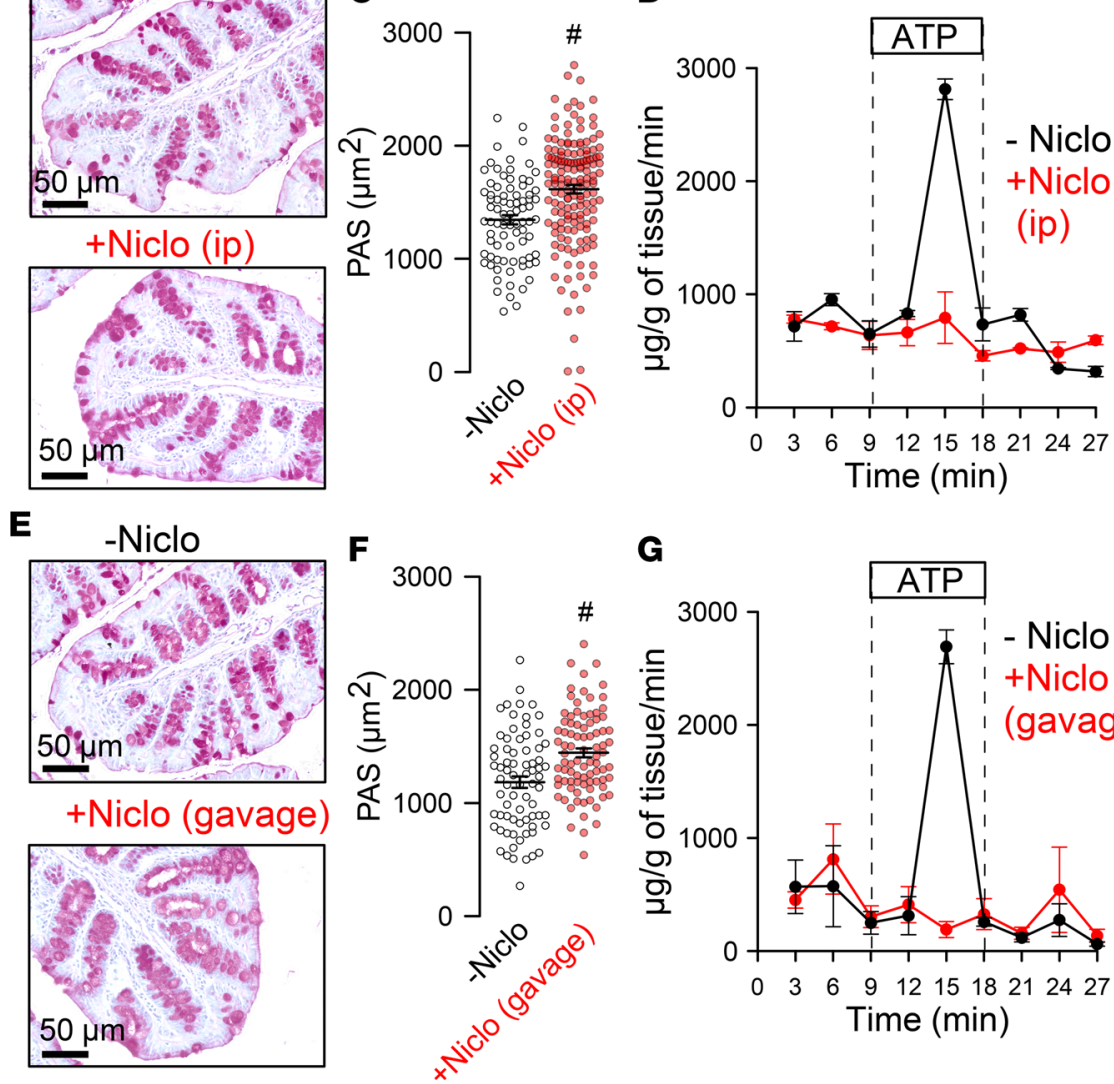

\section{G}

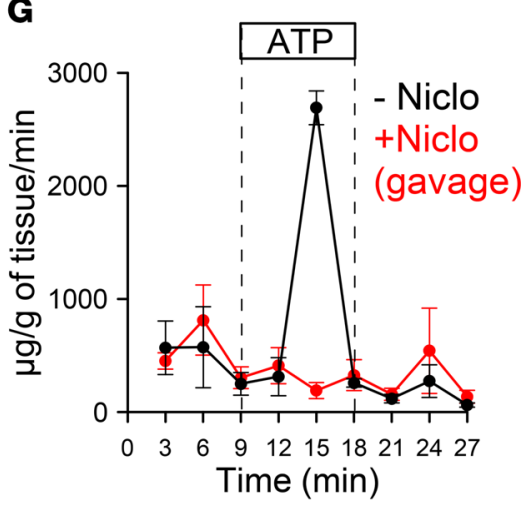

Figure 7. Effect of niclosamide on intestinal mucus release. (A) Acute mucus secretion in excised WT colon activated by MCh or ATP, respectively, and inhibition by acute perfusion with niclosamide (10 $\mu$ M) $(n=3-5$ mice). (B-D) Effect of i.p. injection of niclosamide (10 mM stock dissolved in DMSO) at a concentration of $13 \mathrm{mg} / \mathrm{kg} / \mathrm{d}$ (dissolved in corn oil, total volume $100 \mu \mathrm{L}$ ) on PAS staining ( $n=3$ mice/81-156 sections) (B and $\mathbf{C}$ ) and acute mucus discharge induced by perfusion with $100 \mu \mathrm{M}$ ATP ( $n=3$ mice/74-87 sections) (D). (E-C) Effect of application of niclosamide by gavage $(13 \mathrm{mg} / \mathrm{kg} / \mathrm{d}$ ) on PAS staining (E and $\mathbf{F}$ ) and acute mucus discharge induced by perfusion with $100 \mu \mathrm{M}$ ATP (G) $(n=3-5$ mice). Scale bars: $50 \mu \mathrm{m}$. Data are reported as mean \pm SEM. \#Significant difference when compared with niclosamide (unpaired $t$ test).

by Jason R. Rock (Boston University School of Medicine, Boston, Massachusetts, USA) and by Michael J. Holtzman and Y. Zhang (Washington University School of Medicine, St. Louis, Missouri, USA), respectively. Adult mice aged 8-12 weeks were used for the experiments. KO of TMEM16A in ciliated airway epithelial cells (TMEM16A $A^{A / f l}$ FoxJ1-Cre) has been described elsewhere (16). Generation of mice with a floxed TMEM16F allele was described previously (64). For KO of TMEM16F in ciliated epithelial and intestinal epithelial cells, TMEM16F $F^{\text {l/f }}$ mice were crossed with FoxJ1-Cre and Vil1-Cre mice to

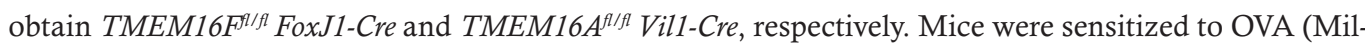
liporeSigma) by i.p. injection of $100 \mu \mathrm{g}$ OVA with $1 \mathrm{mg}$ aluminum hydroxide gel (MilliporeSigma) on days 0 and 14. On days 21, 22, and 23, mice were anesthetized and challenged to OVA by intratracheal 
Table 2. Primers used for RT-PCR

\begin{tabular}{|c|c|c|}
\hline & Accession no. & Primer \\
\hline Tmem16a & NM_178642.5 & $\begin{array}{l}\text { for: 5'-GTGACAACACCTGCAGCTAC } \\
\text { rev: 5'-GCTCCAGCTGTCGAGATTC }\end{array}$ \\
\hline Tmem16f lung & NM_001253813.1 & $\begin{array}{l}\text { for: 5'-ATGCAGATGATGACTAGGAAGG } \\
\text { rev: 5'-CGGAGCACCTTCGTGAACC }\end{array}$ \\
\hline Tmem16f intestine & NM_001253813.1 & $\begin{array}{l}\text { for: 5'-CATACGAATCTAACCTTATCTCC } \\
\text { rev: } 5^{\prime} \text {-CATTCTCTGTACAGGAGGTAAC }\end{array}$ \\
\hline P2rY2 & NM_008773.4 & $\begin{array}{l}\text { for: 5'-GGAACCCTGGAATAGCACC } \\
\text { rev: 5'-CTGGTGGTGACGAAGTAGAG }\end{array}$ \\
\hline Chrm3 & NM_033269.4 & $\begin{array}{l}\text { for: 5'-CTTTTCTATTACCAGGCCACTC } \\
\text { rev: 5'-CCTTCAGTACAATCGAATAGATC }\end{array}$ \\
\hline Gapdh & NM_001289726.1 & $\begin{array}{l}\text { for: 5'-GTATTGGGCGCCTGGTCAC } \\
\text { rev: 5'-CTCCTGGAAGATGGTCATGG }\end{array}$ \\
\hline
\end{tabular}

instillation of $50 \mu \mathrm{g}$ OVA in $100 \mu \mathrm{L}$ saline. Control mice were sham sensitized with saline and aluminum hydroxide gel (MilliporeSigma) and challenged to $100 \mu \mathrm{L}$ saline by intratracheal instillation (65). NFA $(0.5 \mathrm{mg} / \mathrm{kg} / \mathrm{d}$ for 3 days, dissolved in corn oil; MilliporeSigma) and niclosamide ( $13 \mathrm{mg} / \mathrm{kg} / \mathrm{d}$ for 3 days, dissolved in corn oil) were administered via i.p. injections. In addition, niclosamide $(13 \mathrm{mg} / \mathrm{kg} / \mathrm{d}$, dissolved in corn oil) was received via gavage. For control treatment, vehicles were used.

Cell culture, crypt isolation. HEK293 cells were cultured in DMEM (GIBCO) supplemented with 10\% FBS (Capricorn). Calu3 cells were grown in DMEM-F12 (GIBCO) supplemented with 1\% HEPES, 1\% L-glutamine, and 10\% FBS (Capricorn). Cell lines were obtained originally from ATCC and were grown at $37^{\circ} \mathrm{C}$ in the absence of antibiotics in a humidified atmosphere with $5 \% \mathrm{CO}_{2}$. For experiments, cells were grown on glass coverslips and subsequently mounted into a perfused bath on the stage of an inverted microscope (Zeiss, Axiovert 200). Animals were euthanized by $\mathrm{CO}_{2}$ inhalation. Crypts were isolated from mouse colon by incubation of the resected colon in chelating buffer (containing $96 \mathrm{mM} \mathrm{NaCl}, 1,5 \mathrm{mM} \mathrm{KCl}, 10$ mM HEPES-Tris, $27 \mathrm{mM}$ Na-EDTA, $55 \mathrm{mM}$ sorbitol, $44 \mathrm{mM}$ sucrose, $1 \mathrm{mM}$ DTT, $1 \mu \mathrm{M}$ indomethacin) on ice, washed afterward, and mechanically agitated. Crypt-rich supernatant was collected, centrifuged, kept on ice, and used immediately in $\mathrm{Ca}^{2+}$ measurements.

$R T-P C R$, siRNA. RT-PCR was performed using RNA isolated from lungs of WT and TMEM16A- and TMEM16F-KO mice using NucleoSpin RNA columns (Macherey-Nagel). Total RNA (1 $\mu \mathrm{g} / 50 \mu \mathrm{L}$ reaction) was reverse-transcribed using random primer (Promega) and M-MLV Reverse Transcriptase RNAse H Minus (Promega). Each RT-PCR reaction contained sense and antisense primer (Table 2), $0.5 \mu \mathrm{L}$ cDNA, and GoTaq Polymerase (Promega). After 2 minutes at $95^{\circ} \mathrm{C}$ cDNA was amplified for 30 cycles for 30 seconds at $95^{\circ} \mathrm{C}, 30$ seconds at $57^{\circ} \mathrm{C}$, and 1 minute at $72^{\circ} \mathrm{C}$, followed by 10 minutes at $72^{\circ} \mathrm{C}$. PCR products were visualized by loading on peqGREEN (Peqlab, VWR) containing agarose gels and were analyzed using Image (NIH). TMEM16F was downregulated by human siRNA transfection into Calu3 cells using standard methods (lipofectamine, Invitrogen).

Western blotting. Cells (HT29, ATCC HTB-38; Calu-3, ATCC HTB-55) were collected and lysed in 1\% NP-40 lysis buffer (MilliporeSigma) containing protease inhibitor cocktail and DTT (MilliporeSigma). Protein (30-50 $\mu$ g) was separated by $8.5 \%$ SDS-PAGE and transferred to nitrocellulose membranes (Hartenstein). Membranes were blocked with 5\% NFM/TBST (NFM: nonfat milk, A. Hartenstein, catalog A0830,1000; TBST: Tris buffer plus Tween20 [0.1\%], MilliporeSigma) at room temperature for 1 hour and were incubated overnight at $4^{\circ} \mathrm{C}$ with rabbit polyclonal anti-TMEM16F (diluted 1:5000 in 5\% NFM/TBST; Davids Biotechnology) or rabbit polyclonal antiactin (diluted 1:10,000 in 5\% NFM/TBST; MilliporeSigma). Subsequently, membranes were incubated with secondary antibody at room temperature for 2 hours (goat anti-rabbit HRP conjugate, Invitrogen catalog 31460, 1:10000 1\%NFM/TBST). Immunoreactive signals were visualized using SuperSignal chemiluminescence substrate detection kit (Pierce Biotechnology).

IL-8 assay. To measure secretion of the cytokine IL-8 (Peprotech), Calu3 cells were rinsed twice with PBS (Capricorn), placed in OptiMEM (GIBCO), and exposed to LPS (10 $\mu \mathrm{g} / \mathrm{mL}, 48$ hours). After 48 hours, the conditioned medium was collected and used to quantify IL-8 using Quantikine ELISA kits (R\&D Systems) according to the manufacturer's instructions. 
In vitro perfusion of intestine. Mice were sacrificed by cervical dislocation, and excised intestines were placed immediately in ice-cold Ringer's solution and carefully flushed to remove residual luminal contents. The intestinal segments were mounted and perfused vertically in a custom-designed perfusion chamber with a constant temperature, similar to previous methods $(18,66)$. After mounting, the serosal side was exposed to Ringer's solution (120 mM NaCl, $0.4 \mathrm{mM} \mathrm{KH}_{2} \mathrm{PO}_{4}, 1.6 \mathrm{mM} \mathrm{K}_{2} \mathrm{HPO}_{4}, 5 \mathrm{mM}$ D-glucose, $1 \mathrm{mM}$ $\mathrm{MgSO}_{4}, 1.5 \mathrm{mM} \mathrm{Ca}^{2+}$-gluconate, and $25 \mathrm{mM} \mathrm{NaHCO}_{3}$; $\mathrm{pH} 7.4$; continuously gassed with $95 \% \mathrm{O}_{2}$ and $5 \%$ $\mathrm{CO}_{2}$ ) and perfused luminally with glucose-free mannitol-replaced Ringer's solution. Perfusates were collected at a rate of $0.5 \mathrm{~mL} / \mathrm{min}$ at 3-minute intervals for an additional 27 minutes. Tissues were stimulated with MCh $(100 \mu \mathrm{M})$ or ATP $(100 \mu \mathrm{M})$ for 9 minutes, always in the presence of $1 \mu \mathrm{M}$ prostaglandin $\mathrm{E}_{2}$ if not mentioned otherwise. In luminal $\mathrm{Ca}^{2+}$-free solution, $\mathrm{Ca}^{2+}$ was substituted with equimolar EGTA. The amount of mucus secretion was analyzed using a PAS assay (MilliporeSigma). In brief, $50 \mathrm{mM}$ DTT was pipetted into each sample and incubated for 1 hour at $37^{\circ} \mathrm{C}$ under continuous shaking. Then, $0.2 \mathrm{~mL}$ periodic acid $(0.1 \%)$ was added and incubated for 2 hours at $37^{\circ} \mathrm{C}$ and an additional 30 minutes at $20^{\circ} \mathrm{C}$ after $0.2 \mathrm{~mL}$ Schiff's reagent was added (MilliporeSigma). Samples were centrifuged at $500 \mathrm{~g}$ for 5 minutes, and $\mathrm{OD}$ of the resulting solution was measured at $540 \mathrm{~nm}$. The amount of mucus per tissue and minute was calculated using a calibration curve from defined pig gastric mucin (MilliporeSigma).

Histology, mucus staining by Alcian blue and PAS, and quantification. Mice were sacrificed by cervical dislocation. Small and large intestines were removed and cut into 2 sections. One section was kept in Ringer's solution; the other section was exposed to $100 \mu \mathrm{M}$ ATP (ROTH) or $100 \mu \mathrm{M}$ CCH (MilliporeSigma). Before and after each experiment, intestinal sections were fixed for further histological analyses. Mouse airways were fixed by transcardial fixation and were embedded in paraffin or frozen in liquid $\mathrm{N}_{2}$. For paraffin sections, tissues were fixed in $4 \%$ paraformaldehyde, $0.2 \%$ picric acid, and 3.4\% sucrose in PBS and were washed in methanol before embedding in paraffin. For mucus analysis, sections were stained according to standard PAS or Alcian blue methods and examined by light microscopy. A minimum of 9 random images for each embedded tissue were acquired from a minimum of 3 animals (TMEM16A $A^{f / f l}, T M E M 16 F^{\ell / f l}, T M E M 16 A^{f / f l}$ FoxJ1-Cre,

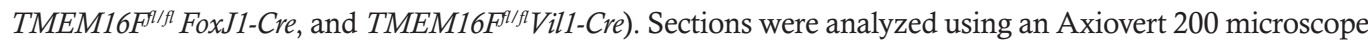
equipped with AxioCam ICc 1 and ApoTome (Zeiss) and analyzed using AxioVision (Zeiss).

Patch clamping. Crypts from jejunum and distal colon were isolated in $\mathrm{Ca}^{2+}$-free Ringer's solution and immobilized on polylysine-coated glass coverslips. HEK293 cells were grown on glass-coated coverslips and were mounted on the stage of an inverted microscope (Zeiss). Experimental procedures were described in detail previously (16).

Measurement of intracellular $\mathrm{Ca}^{2+}$ concentration. All measurements of intracellular $\mathrm{Ca}^{2+}$ concentration of cell lines and isolated goblet cells were performed using the $\mathrm{Ca}^{2+}$-sensitive dye Fura2-AM (Tocris Bioscience), as described earlier (29).

YFP quenching. Quenching of the intracellular fluorescence generated by the iodide-sensitive enhanced YFP was used to measure anion conductance. YFP fluorescence was excited at $490 \mathrm{~nm}$ using a semiautomatic Novostar plate reader (BMG-Labtech). $\mathrm{I}^{-}$influx was induced by replacing $20 \mathrm{mM}$ extracellular $\mathrm{Cl}^{-}$with $20 \mathrm{mM} \mathrm{I}^{-}$in the presence of $1 \mu \mathrm{M}$ ionomycin (Enzo Life Sciences, Enzo Biochem). Background fluorescence was subtracted and autofluorescence was negligible. Changes in fluorescence induced by $\mathrm{I}^{-}$are expressed as initial rates of fluorescence decrease (AU/s) and were analyzed using MARS data-analyzing program (BMG-Labtech).

Materials. All compounds used were of highest available grade of purity: niclosamide, Eact, OVA, MCh, NFA, dichlorophene, (MilliporeSigma). Niclosamide-ETHO, nitazoxanide, tizoxanide (Cayman Chemical), CaCCinhAO1 (Tocris Bioscience), IL-8, and IL-13 (Peprotech). Antibodies were purchased from Thermo Fisher Scientific (TMEM16F; PA5-35240), Bioss (MUC5AC; bs-7166R), and Novus (MUC5B; 6F10-E4). All gene symbols are consistent with Entrez Gene nomenclature, are italicized, and are capitalized according to biological species.

Statistics. Data are reported as mean \pm SEM. Two-tailed Student's $t$ test (for paired or unpaired samples as appropriate) or 1-way ANOVA was used for statistical analysis. A $P$ value less than 0.05 was accepted as a significant difference.

Study approval. All animal experiments complied with the ARRIVE guidelines and were carried out in accordance with the UK Animals (Scientific Procedures) Act 1986 and associated guidelines, as well as EU Directive 2010/63/EU for animal experiments. The study was approved by the local Ethics Committee of the Government of Unterfranken, RUF-55.2.2-2532-2-677-19; approval on August 24, 2018. 


\section{Author contributions}

$\mathrm{KK}, \mathrm{RB}, \mathrm{RS}$, and IC conceived the project. RS, RB, and IC performed the experimental work. All authors analyzed the data and prepared figures. $\mathrm{KK}, \mathrm{RS}, \mathrm{RB}$, and IC wrote the manuscript and all authors edited the manuscript. KK supervised all aspects of the project.

\section{Acknowledgments}

This study was supported by Cystic Fibrosis Trust SRC 013, Gilead Stiftung, DFG KU756/14-1, and DFG - 387509280 SFB 1350. The excellent technical assistance by B. Wild, P. Seeberger, and E. Tartler is gratefully acknowledged. We gratefully acknowledge the gift of mice with a floxed TMEM16A allele by Jason $\mathrm{R}$. Rock and of the FoxJ1-Cre mice by Michael J. Holtzman and Y. Zhang.

1. Boucher RC. Airway surface dehydration in cystic fibrosis: pathogenesis and therapy. Annu Rev Med. 2007;58:157-170

2. Stoltz DA, Meyerholz DK, Welsh MJ. Origins of cystic fibrosis lung disease. N Engl J Med. 2015;372(4):351-362.

3. Huang F, et al. Calcium-activated chloride channel TMEM16A modulates mucin secretion and airway smooth muscle contraction. Proc Natl Acad Sci U S A. 2012;109(40):16354-16359.

4. Kondo M, et al. Chloride ion transport and overexpression of TMEM16A in a guinea-pig asthma model. Clin Exp Allergy. 2017;47(6):795-804.

5. Fahy JV, Dickey BF. Airway mucus function and dysfunction. N Engl J Med. 2010;363(23):2233-2247.

6. Huang F, et al. Studies on expression and function of the TMEM16A calcium-activated chloride channel. Proc Natl Acad Sci U S A. 2009;106(50):21413-21418.

7. Gallos G, et al. Functional expression of the TMEM16 family of calcium-activated chloride channels in airway smooth muscle. Am J Physiol Lung Cell Mol Physiol. 2013;305(9):L625-L634.

8. Danielsson J, et al. Antagonists of the TMEM16A calcium-activated chloride channel modulate airway smooth muscle tone and intracellular calcium. Anesthesiology. 2015;123(3):569-581.

9. Kunzelmann K, Schreiber R, Hadorn HB. Bicarbonate in cystic fibrosis. J Cyst Fibros. 2017;16(6):653-662.

10. Pezzulo AA, et al. Reduced airway surface $\mathrm{pH}$ impairs bacterial killing in the porcine cystic fibrosis lung. Nature. 2012;487(7405):109-113.

11. Schultz A, et al. Airway surface liquid $\mathrm{pH}$ is not acidic in children with cystic fibrosis. Nat Commun. 2017;8(1):1409.

12. Scudieri P, et al. Increased expression of ATP12A proton pump in cystic fibrosis airways. JCI Insight. 2018;3(20):123616.

13. Colombo JL. Long-acting bronchodilators in cystic fibrosis. Curr Opin Pulm Med. 2003;9(6):504-508.

14. Matusovsky OS, et al. Contractile properties of intrapulmonary airway smooth muscle in cystic fibrosis. Am J Respir Cell Mol Biol. 2019;60(4):434-444.

15. Pascoe CD, Halayko AJ. Shot down inflamed: airway smooth muscle bronchodilator insensitivity in cystic fibrosis. Am J Respir Cell Mol Biol. 2019;60(4):379-381.

16. Benedetto R, et al. Epithelial chloride transport by CFTR requires TMEM16A. Sci Rep. 2017;7(1):12397.

17. Papp R, et al. Targeting TMEM16A to reverse vasoconstriction and remodelling in idiopathic pulmonary arterial hypertension. Eur Respir J. 2019;53(6):1800965.

18. Benedetto R, Cabrita I, Schreiber R, Kunzelmann K. TMEM16A is indispensable for basal mucus secretion in airways and intestine. FASEB J. 2019;33(3):4502-4512.

19. Caci E, et al. Upregulation of TMEM16A protein in bronchial epithelial cells by bacterial pyocyanin. PLoS One. 2015;10(6):e0131775.

20. Wang $\mathrm{P}$, et al. Inflammatory mediators mediate airway smooth muscle contraction through a G protein-coupled receptor-transmembrane protein 16A-voltage-dependent $\mathrm{Ca}^{2+}$ channel axis and contribute to bronchial hyperresponsiveness in asthma. $J$ Allergy Clin Immunol. 2018;141(4):1259-1268.e11.

21. Miner K, et al. Drug Repurposing: The Anthelmintics Niclosamide and Nitazoxanide Are Potent TMEM16A Antagonists That Fully Bronchodilate Airways. Front Pharmacol. 2019;10:51.

22. Forrest AS, et al. Increased TMEM16A-encoded calcium-activated chloride channel activity is associated with pulmonary hypertension. Am J Physiol, Cell Physiol. 2012;303(12):C1229-C1243.

23. Wells JM, et al. Pulmonary artery enlargement and cystic fibrosis pulmonary exacerbations: a cohort study. Lancet Respir Med. 2016;4(8):636-645.

24. Harkness LM, Kanabar V, Sharma HS, Westergren-Thorsson G, Larsson-Callerfelt AK. Pulmonary vascular changes in asthma and COPD. Pulm Pharmacol Ther. 2014;29(2):144-155.

25. Benedetto R, et al. Plasma membrane-localized TMEM16 proteins are indispensable for expression of CFTR. JMol Med. 2019;97(5):711-722.

26. Kunzelmann K, et al. Airway epithelial cells - functional links between CFTR and anoctamin dependent $\mathrm{Cl}^{-}$secretion. Int $J$ Biochem Cell Biol. 2012;44(11):1897-1900.

27. Nakano T, et al. Niflumic acid suppresses interleukin-13-induced asthma phenotypes. Am J Respir Crit Care Med. 2006;173(11):1216-1221.

28. Tian Y, et al. Control of TMEM16A by INO-4995 and other inositolphosphates. Br J Pharmacol. 2013;168(1):253-265.

29. Cabrita I, et al. Differential effects of anoctamins on intracellular calcium signals. FASEB J. 2017;31(5):2123-2134.

30. Namkung W, Yao Z, Finkbeiner WE, Verkman AS. Small-molecule activators of TMEM16A, a calcium-activated chloride channel, stimulate epithelial chloride secretion and intestinal contraction. FASEB J. 2011;25(11):4048-4062.

31. Danielsson J, Mikami M, Emala CW. Antagonism of the TMEM16A calcium-activated chloride channel attenuates allergic 
lung inflammation. Am J Respir Crit Care Med. 2017;195:A5290.

32. Liang L, et al. Inhibitory effects of niclosamide on inflammation and migration of fibroblast-like synoviocytes from patients with rheumatoid arthritis. Inflamm Res. 2015;64(3-4):225-233.

33. Chen Y, Thai P, Zhao YH, Ho YS, DeSouza MM, Wu R. Stimulation of airway mucin gene expression by interleukin (IL)-17 through IL-6 paracrine/autocrine loop. J Biol Chem. 2003;278(19):17036-17043.

34. Sirianant L, Ousingsawat J, Wanitchakool P, Schreiber R, Kunzelmann K. Cellular volume regulation by anoctamin 6: Ca ${ }^{2+}$, phospholipase A2 and osmosensing. Pflugers Arch. 2016;468(2):335-349.

35. Wanitchakool P, et al. Cellular defects by deletion of ANO10 are due to deregulated local calcium signaling. Cell Signal. 2017;30:41-49.

36. Bricogne $\mathrm{C}$, et al. TMEM16F activation by $\mathrm{Ca}^{2+}$ triggers plasma membrane expansion and directs PD-1 trafficking. Sci Rep . 2019;9(1):619.

37. Chen W, Mook RA, Premont RT, Wang J. Niclosamide: beyond an antihelminthic drug. Cell Signal. 2018;41:89-96.

38. Kunzelmann K, Ousingsawat J, Benedetto R, Cabrita I, Schreiber R. Contribution of anoctamins to cell survival and cell death. Cancers (Basel). 2019;11(3):E382.

39. Pedemonte N, Galietta LJ. Structure and function of TMEM16 proteins (anoctamins). Physiol Rev. 2014;94(2):419-459.

40. Jha A, et al. Anoctamin 8 tethers endoplasmic reticulum and plasma membrane for assembly of $\mathrm{Ca}^{2+}$ signaling complexes at the ER/PM compartment. EMBO J. 2019;38(12):e101452.

41. Walker NM, Simpson JE, Levitt RC, Boyle KT, Clarke LL. Talniflumate increases survival in a cystic fibrosis mouse model of distal intestinal obstructive syndrome. J Pharmacol Exp Ther. 2006;317(1):275-283.

42. Scudieri P, et al. Association of TMEM16A chloride channel overexpression with airway goblet cell metaplasia. J Physiol (Lond). 2012;590(23):6141-6155.

43. Mazzone A, et al. Identification and characterization of a novel promoter for the human ANO1 gene regulated by the transcription factor signal transducer and activator of transcription 6 (STAT6). FASEB J. 2015;29(1):152-163.

44. Qin Y, Jiang Y, Sheikh AS, Shen S, Liu J, Jiang D. Interleukin-13 stimulates MUC5AC expression via a STAT6-TMEM16AERK1/2 pathway in human airway epithelial cells. Int Immunopharmacol. 2016;40:106-114.

45. Schreiber R, et al. Expression and function of epithelial anoctamins. J Biol Chem. 2010;285(10):7838-7845.

46. Hammer C, et al. A coding variant of ANO10, affecting volume regulation of macrophages, is associated with Borrelia seropositivity. Mol Med. 2015;21:26-37.

47. Schreiber R, et al. Lipid peroxidation drives renal cyst growth in vitro through activation of TMEM16A. JAm Soc Nephrol. 2019;30(2):228-242.

48. Jin X, et al. Activation of the Cl-channel ANO1 by localized calcium signals in nociceptive sensory neurons requires coupling with the IP3 receptor. Sci Signal. 2013;6(290):ra73.

49. Kunzelmann K, et al. Modulating Ca ${ }^{2+}$ signals: a common theme for TMEM16, Ist2, and TMC. Pflugers Arch. 2016;468(3):475-490

50. Ousingsawat J, et al. Anoctamin 6 mediates effects essential for innate immunity downstream of P2X7 receptors in macrophages. Nat Commun. 2015;6:6245

51. Yang $\mathrm{H}$, et al. TMEM16F forms a $\mathrm{Ca}^{2+}$-activated cation channel required for lipid scrambling in platelets during blood coagulation. Cell. 2012;151(1):111-122.

52. He M, Ye W, Wang WJ, Sison ES, Jan YN, Jan LY. Cytoplasmic $\mathrm{Cl}^{-}$couples membrane remodeling to epithelial morphogenesis. Proc Natl Acad Sci U S A. 2017;114(52):E11161-E11169.

53. Mall MA, Galietta LJ. Targeting ion channels in cystic fibrosis. J Cyst Fibros. 2015;14(5):561-570

54. Ruffin M, et al. Anoctamin 1 dysregulation alters bronchial epithelial repair in cystic fibrosis. Biochim Biophys Acta. 2013;1832(12):2340-2351

55. Mall M, et al. Modulation of $\mathrm{Ca}^{2+}$-activated $\mathrm{Cl}^{-}$secretion by basolateral $\mathrm{K}^{+}$channels in human normal and cystic fibrosis airway epithelia. Pediatr Res. 2003;53(4):608-618.

56. Billet A, Hanrahan JW. The secret life of CFTR as a calcium-activated chloride channel. J Physiol (Lond). 2013;591(21):5273-5278

57. Moss RB. Pitfalls of drug development: lessons learned from trials of denufosol in cystic fibrosis. J Pediatr. 2013;162(4):676-680

58. Ratjen F, et al. Long term effects of denufosol tetrasodium in patients with cystic fibrosis. J Cyst Fibros. 2012;11(6):539-549.

59. Imperi F, et al. New life for an old drug: the anthelmintic drug niclosamide inhibits Pseudomonas aeruginosa quorum sensing Antimicrob Agents Chemother. 2013;57(2):996-1005.

60. Costabile G, et al. Toward repositioning niclosamide for antivirulence therapy of Pseudomonas aeruginosa lung infections: development of inhalable formulations through nanosuspension technology. Mol Pharm. 2015;12(8):2604-2617.

61. Gwisai T, et al. Repurposing niclosamide as a versatile antimicrobial surface coating against device-associated, hospital-acquired bacterial infections. Biomed Mater. 2017;12(4):045010.

62. Morin F, Kavian N, Nicco C, Cerles O, Chéreau C, Batteux F. Improvement of sclerodermatous graft-versus-host disease in mice by niclosamide. J Invest Dermatol. 2016;136(11):2158-2167.

63. Li Y, Li PK, Roberts MJ, Arend RC, Samant RS, Buchsbaum DJ. Multi-targeted therapy of cancer by niclosamide: a new application for an old drug. Cancer Lett. 2014;349(1):8-14.

64. Schenk LK, Ousingsawat J, Skryabin BV, Schreiber R, Pavenstädt H, Kunzelmann K. Regulation and function of TMEM16F in renal podocytes. Int J Mol Sci. 2018;19(6):E1798.

65. Schreiber R, Castrop H, Kunzelmann K. Allergen-induced airway hyperresponsiveness is absent in ecto-5'-nucleotidase (CD73)-deficient mice. Pflugers Arch. 2008;457(2):431-440.

66. Garcia MA, Yang N, Quinton PM. Normal mouse intestinal mucus release requires cystic fibrosis transmembrane regulator-dependent bicarbonate secretion. J Clin Invest. 2009;119(9):2613-2622. 